\title{
Simulation of the Core Technology of a Greenhouse-Monitoring System Based on a Wireless Sensor Network
}

\author{
http://dx.doi.org/10.3991/ijoe.v12i05.5735 \\ Yuhong Zhou, Yunfang Xie and Limin Shao \\ Agricultural University of Hebei, Baoding, Hebei, China
}

\begin{abstract}
To solve the topology structure of a greenhouse environment-monitoring system and the ductility of joints, this study presents a design of a greenhouse-monitoring system based on the ZigBee wireless sensor network (WSN). The hardware and software designs of the network node are provided, and the process of the ZigBee network coordinator is elucidated. The system uses the microcontroller unit PIC18F4620CC2420 wireless transceiver module to send and receive data. Data from temperature and humidity sensors are collected using an inter-integrated circuit bus through the ZigBee network transmission to the monitoring platform. Test results show that the system has the advantages of having a simple structure, flexible nodes, and low power consumption. It can effectively monitor the temperature and humidity in a wireless environment. WSNs considerably help in greenhouse environment monitoring. The use of advanced technology to control greenhouse temperature and humidity can satisfy the optimum growth environment of greenhouse crops and effectively improve the yield and quality of crops. The application of WSNs in greenhouse monitoring is significant to the development of modern and precision agriculture in China.
\end{abstract}

Index Terms-Greenhouse monitoring, Heterogeneous Network, WSN

\section{INTRODUCTION}

With the increase in population and the decrease in arable land, realizing the modernization of agriculture and its sustainable development is necessary.

Most greenhouses still use artificial management and mainly rely on artificial experience, with lack of systematic scientific guidance. Their application of science and technology is limited, and they adopt only artificial moisturizing and insulation. The reasonable distribution of various elements, such as temperature, gas, fertilizer, light, etc., cannot be predicted, calculated, and controlled, which hinders the high rate and quality of greenhouses.

A wireless sensor network (WSN) has considerable microcomputing power and can collect data via wireless communication and nodes through a specific communication protocol to achieve data exchange. WSN is one of the 10 most influential technologies in the world in the $21 \mathrm{st}$ century according to the United States.

Greenhouse-monitoring technology, which comprises an automatic controller, a computer network, sensors, and other technologies, is used in agriculture to precisely monitor and control greenhouse temperature, humidity, light, nutrients, and other elements. The corresponding sensor nodes can be placed in an area to be detected, and the network is composed of the corresponding sensor nodes.

The United States Department of Defense Vision Research Program has provided funds to Harvard University, the Massachusetts Institute of Technology, and other famous universities to assist in the study of WSNs [1]. In 2003, the National Natural Science Foundation of the United States invested $\$ 100$ million to develop a sensor network research program [2]. Research on WSN in China began in the late 1990 s, with foreign basic synchronization. Since 2003, the National Natural Science Foundation of China has established key technology research projects related to sensor networks [3]. From 2005, long-term strategic development plans have been formulated for WSN combined with other technologies [4].

At present, greenhouse-monitoring and control systems with WSNs remain within a small-scale trial stage. A greenhouse measurement and control system based on WSN is constructed, and remote monitoring and control system is realized via General Packet Radio Service (GPRS) or the Internet. Compared with monitoring and control systems based on WSNs, the wired mode presents several problems, such as higher cost, wiring complexity, and more fault points. Therefore, monitoring and control system based on WSN is the future development direction of greenhouse-monitoring and control systems. Research on the application of WSN in greenhouse monitoring is of highly significant to the modernization of the country.

The remainder of this paper is organized as follows. Section 2 discusses research on WSNs. Section 3 presents the design of the proposed system hardware. Section 4 describes the system performance test and the result analysis. Section 5 concludes the study.

\section{STATE OF THE ART}

\section{A. ZigBee Technology}

ZigBee technology has a simple structure, low cost, low power consumption, low rate, and short-distance wireless communication technology. ZigBee was previously known as "Lite HomeRF," "RF-EasyLink," or "FireFly" wireless technology. The characteristics of the ZigBee protocol are listed in Table I.

\section{B. ZigBee Protocol Framework}

The ZigBee protocol stack defines four layers, namely, physical layer (PHY), media access control layer (MAC), 
network layer (NWK), and application layer (APL). The frame diagram is shown in Figure 1, in which PHY and MAC adopt the IEEE802.15.4 protocol standard and the NWK and APL frameworks are designed by the ZigBee alliance $[5,6]$.

PHY ZigBee is at the bottom of the PHY protocol and uses the standard IEEE802.15.4 protocol. ZigBee uses 2.4 $\mathrm{GHz}$ and $915 / 868 \mathrm{MH}$. The three free bands of ZigBee have a total of 27 physical channels; the $866 \mathrm{MHz}$ band has only 1 channel; the $915 \mathrm{MHz}$ band has 10 channels, with a band gap of $2 \mathrm{MHz}$; and the $2.4 \mathrm{GHz}$ band has 16 channels, with an interval of $5 \mathrm{MHz}$, as shown in Table 2. MAC is also defined by the main function of data packet segmentation and reorganization to ensure that the data packets can be transmitted in accordance with the order in the network and the receiver back to the original data. NWK mainly receives APL and the data packet and combines the frame header to MAC transmission. If the data packet of a node is received, then this data packet analysis is concurrent to APL. Otherwise, the packet is sent to the subsequent routing node to build a new network or join an existing network, to maintain the network neighbor table, and to control the receiving time and status of the receiver. ZigBee APL is mainly composed of the application framework, the application support sublayer, and the ZigBee device object. APL provides the interface and the corresponding application object, and the user can implement them according to their own needs [7].

\section{ZigBee Network Topology}

The ZigBee protocol supports multiple network topologies, namely, tree, star, and mesh networks. A star network is based on a coordinated node as the center plus a number of terminal nodes. In this structure, all data are processed through the coordination of nodes. Three disadvantages are determined, namely, the entire network will be paralyzed when nodes are not coordinated, the corresponding additional load is high, and network obstruction occurs [9].

\section{Brief Introduction into Embedded Web}

In practical applications, embedded web technology has the following architectures [10].

(1) Direct embedded network: This network generally uses a 32-bit or 64-bit microcontroller, adds the corresponding network interface and software, and finally, is realized and connected. However, the direct network will occupy a huge amount of system resources and affect its flexibility.

(2) Combined embedded system and network protocol chip network: The network protocol is integrated into the network chip and then connected to the Internet with the subcarrier multiplexing input/output port. The network reaction speed is accordingly fast, but the hardware cost is high.

(3) Based on embedded gateway network: The embedded system and gateway use the traditional RS-232, RS422 , and controller area network bus connection. The embedded gateway provides the conversion of a heterogeneous network to achieve the traditional structure of network and transmission control protocol (TCP)/Internet protocol (IP) data exchange to access the Internet. Its main characteristic is to achieve data exchange between multiple protocols and TCP/IP.
TABLE I.

Protocol CHARACTERISTICS OF ZIGBEE

\begin{tabular}{|l|l|}
\hline Characteristic & High-Power LED Feature Narration \\
\hline $\begin{array}{l}\text { Low power con- } \\
\text { sumption }\end{array}$ & $\begin{array}{l}\text { The MAC protocol of the ZigBee layer uses } \\
\text { the talk-when-ready collision avoidance } \\
\text { mechanism. }\end{array}$ \\
\hline High data reliability & $\begin{array}{l}\text { In the ZigBee protocol network, each coordi- } \\
\text { nation point can be connected to 255 nodes, } \\
\text { which can be connected to the routing node. }\end{array}$ \\
\hline Compatibility & $\begin{array}{l}\text { The ZigBee protocol can be compatible with } \\
\text { existing network standards. The CSMACA } \\
\text { protocol is used for channel access. }\end{array}$ \\
\hline Security & $\begin{array}{l}\text { The ZigBee protocol provides authentication } \\
\text { and data integrity detection, and the AES-128 } \\
\text { algorithm can be used for encryption. }\end{array}$ \\
\hline $\begin{array}{l}\text { Low cost of imple- } \\
\text { mentation }\end{array}$ & $\begin{array}{l}\text { The protocol has a simple storage space, low } \\
\text { cost, and no patent fee. }\end{array}$ \\
\hline
\end{tabular}

TABLE II.

Protocol CHARACTERISTICS OF ZigBEE

\begin{tabular}{|l|l|l|l|l|}
\hline $\begin{array}{l}\text { Channel } \\
\text { number }\end{array}$ & $\begin{array}{l}\text { Center } \\
\text { frequency }\end{array}$ & $\begin{array}{l}\text { Channel } \\
\text { spacing }\end{array}$ & $\begin{array}{l}\text { Upper } \\
\text { frequency }\end{array}$ & $\begin{array}{l}\text { Lower } \\
\text { frequency }\end{array}$ \\
\hline $\mathrm{x}=0$ & 868.3 & 1 & 868.6 & 868.0 \\
\hline $\begin{array}{l}\mathrm{x}= \\
1,2,3, \ldots, 10\end{array}$ & $906+2(\mathrm{x}-1)$ & 2 & 928.0 & 902.0 \\
\hline $\begin{array}{l}\mathrm{x}= \\
11,12, \ldots, 26\end{array}$ & $2405+5(\mathrm{x}-11)$ & 5 & 2483.5 & 2400.0 \\
\hline
\end{tabular}

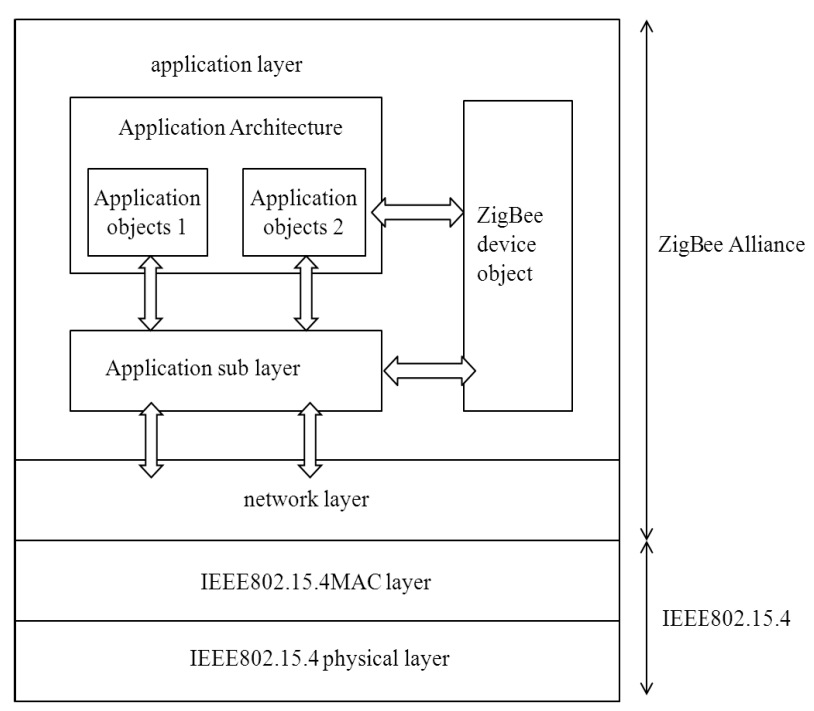

Figure 1. Platform design structure

\section{MEthodology}

\section{A. Sensor Design}

Sensors are a standing wave ratio method for measuring soil water content. Soil water is the main ingredient, with solid and air. The relative dielectric constant of air and solid is considerable, and soil impedance is mainly determined by soil water. Topp confirmed the soil dielectric constant and volume of soil water rate in a single deterministic value polynomial relationship, as shown in Formula 1:

$$
\varepsilon=3.03+9.3 \theta_{v}+146 \theta_{v}^{2}-76.7 \theta_{v}^{3}
$$


The sensor is mainly composed of a high-frequency signal-generating circuit, a transmission line equivalent circuit network, a four-needle probe, a detection circuit, an op amp differential amplifier circuit, and other processing circuit composition. To produce a high-frequency sine wave signal using a crystal oscillator, the high-frequency signal is transmitted through the center probe in the soil after the coaxial transmission line. Electromagnetic fields exist in the soil medium between the probe and the external probe. When the sensor probe is inserted into the soil, the sine wave signal is transmitted through the soil medium around the probe. A part of the electromagnetic wave occurs in the probe and the transmission line equivalent network circuit connection because of the mismatch between the transmission line equivalent network circuit and the probe impedance. In the transmission line equivalent network circuit, the incident wave and reflected wave superposition forms a standing wave. The terminal reflection coefficient formula is derived from transmission line 2.

$$
\Gamma_{2}=\left|\Gamma_{2}\right| e^{j \varphi 2}
$$

The reflection coefficient from terminal $\mathrm{Z}$ is

$$
\Gamma_{Z}=\left|\Gamma_{2}\right| e^{j\left(\varphi_{2}-2 \beta z\right)}
$$

The voltage at the end of $\mathrm{Z}$ after the formation of the standing wave is the superposition of the incident and reflected waves.

$$
U(z)=U_{0}\left(1+\left|\Gamma_{2}\right| e^{j\left(\varphi_{2}-2 \beta z\right)}\right)
$$

When a quarter wavelength transmission line has an equivalent length, the terminal reflection coefficient presents no phase shift. Traveling and standing waves occur at the transmission line terminal and the transmission line at the other end. The output voltage difference from the ends is the largest.

The transmission line terminal voltage is

$$
U_{1}=U_{0}\left(1+\left|\Gamma_{2}\right| e^{j \varphi_{2}}\right)
$$

The voltage from terminal $\lambda / 4$ is

$$
U_{2}=U_{0}\left(1+\left|\Gamma_{2}\right| e^{j\left(\varphi_{2}-\pi\right)}\right)=U_{0}\left(1-\left|\Gamma_{2}\right| e^{j \varphi_{2}}\right)
$$

Therefore, the voltage difference between the two ends of the transmission line is

$$
U_{2}=U_{0}\left(1+\left|\Gamma_{2}\right| e^{j\left(\varphi_{2}-\pi\right)}\right)=U_{0}\left(1-\left|\Gamma_{2}\right| e^{j \varphi_{2}}\right)
$$

where $Z_{c}$ is the characteristic impedance of the transmission line, $Z_{\mathrm{L}}$ is the characteristic impedance of the soil moisture sensor probe, and $U_{O}$ is the high frequency

The oscillator (crystal) amplitude is constant. The design of the soil moisture content sensor is based on the principle of standing wave ratio.

\section{B. Effects of ZigBee Terminal and Sink Nodes}

The ZigBee terminal node includes a sensor, a candidate to imitate block, a power module, and a communication module. The ZigBee sink node includes a GPRS data terminal unit (DTU), a controller module, a power module, and a communication module.

A high-frequency signal requires a $3.3 \mathrm{~V}$ supply voltage. The differential amplifier circuit and operational amplifier have $5 \mathrm{~V}$ and $5 \mathrm{~V}$ dual-power supply. In view of this situation, the sensor comprises linear low-voltage power conversion chips $\operatorname{lm} 1117-3.3$ and $1 \mathrm{p} 2950$, and converts the output voltage of lithium battery from $9 \mathrm{~V}$ to 3.3 $\mathrm{V}$ and $5 \mathrm{~V}$. The icl7660 negative-pressure conversion chip can convert the voltage from $+5 \mathrm{~V}$ to $-5 \mathrm{~V}$ for the operational amplifier power.

This sensor uses a crystal oscillator and produces a 100 $\mathrm{MHz}$ high-frequency signal. The mismatch in the transmission line equivalent circuit network requires adding capacitance, inductance, resistance, etc. These factors form a matching network of the oscillator output and transmission line input, thereby reducing the reflected signal.

The light intensity conversion voltage circuit converts the extremely weak current signal generated by the silicon photovoltaic cell into an output voltage ranging from $0 \mathrm{~V}$ to $5 \mathrm{~V}$, which is convenient for the subsequent signal processing. The voltage range conversion circuit converts the $0 \mathrm{~V}$ to $5 \mathrm{~V}$ voltage signal to the $0.4 \mathrm{~V}$ to $2 \mathrm{~V}$ voltage signal. The voltage transfer circuit current implements 0.4 $\mathrm{V}$ to $2 \mathrm{~V}$ voltage into $4 \mathrm{~mA}$ to $20 \mathrm{~mA}$ current signal. The regulated power supply-generating circuit actually uses 4 $\mathrm{mA}$ to $20 \mathrm{~mA}$ current generated in front of the light intensity of illumination turn voltage circuit. The voltage conversion circuit provides stable power supply voltage. The circuit contains a current source, which can reduce the external connected power supply voltage variation that affects transmitter performance.

The circuit mainly includes the microcontroller unit (MCU) RS-232, STM8S103-level conversion chip SP3222, and RS-485-level conversion chip MAX485. Signal is inputted into the microcontroller after using a single-chip internal analog-to-digital conversion module, SP3222, and MAX485. The signal is converted into the corresponding standard output signal.

To reduce the complexity of system design, air temperature and humidity sensors, carbon dioxide sensors, and GPRS DTU use commercial equipment. This system selects the new generation digital temperature and humidity sensor of Swiss SHT71 from Sensirion. The selected sensor has the following advantages: high precision, fast response time, programmable measurement resolution, low power consumption, and strong anti-interference capability. Its relative humidity accuracy is $+3 \%$, and the temperature is $0.40{ }^{\circ} \mathrm{C}$.

The sht71 digital sensor has an inter-integrated circuit bus digital interface, cyclic redundancy check, and digital transmission check. It can conveniently and directly connect to other microcontroller systems, increase the development of sensor interfaces, and provide convenience and reliability.

The main controller module chip uses Carl MC9S08GT16 MCU and contains a sensor and a ZigBee communication module and interface. Other specific modules in the design or selection have been previously 
introduced. Figure 2 presents the ZigBee terminal nodes of the physical map, and Figure 4 shows the ZigBee sink node of the physical map.

The H-550 carbon dioxide sensor is utilized; it has the advantages of small volume, light weight, module in a reserved easy plug pin, and convenient connection with other MCU and equipment. The H-550 sensor comprises USART and C II, which makes transferring and reading data easy. This sensor is suitable for various occasions.

This system utilizes the GPRS DTU of ZHDIX, which is built in simcom and GPRS $\operatorname{sim} 300 \mathrm{z}$ module, and the GPRS network is the communication platform. The GPRS DTU provides the standard RS-232RS-485 and TTL, as well as three other types of interface. The system can be directly connected with MCU and other equipment. Transparent communication enables the non-IP system equipment to easily achieve GPRS network and Internet access through the product.

\section{RESUlt ANALYSIS AND DiscusSiON}

The outputs of the soil moisture sensor and the temperature and humidity sensor used in this system are all voltage signals. The sensors need to be calibrated, such that the quantitative relationship between the output voltage of the sensors and the measured parameters can be obtained accurately. The soil moisture sensor is calibrated by the most authoritative soil-drying method in determining soil moisture. To calibrate the dry red soil in the Yun-

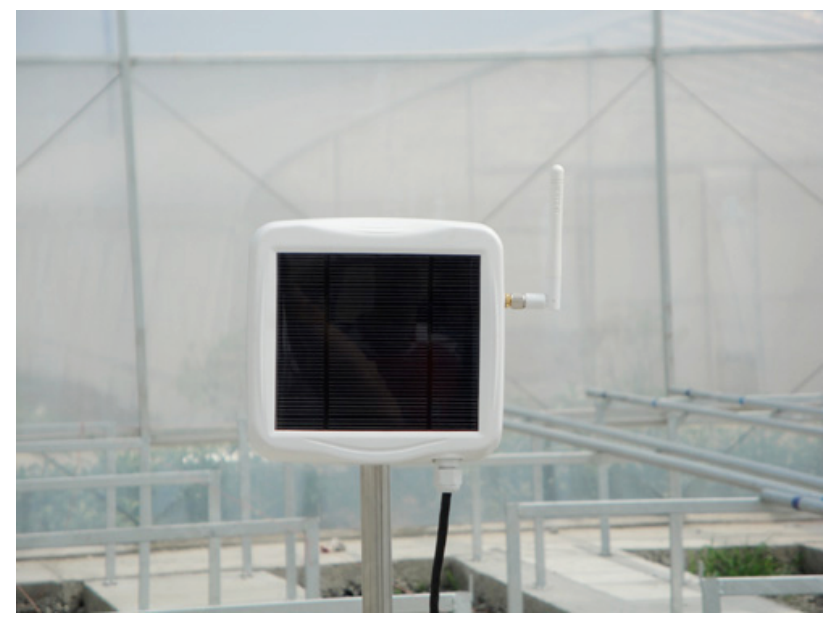

Figure 2. ZigBee terminal node graph

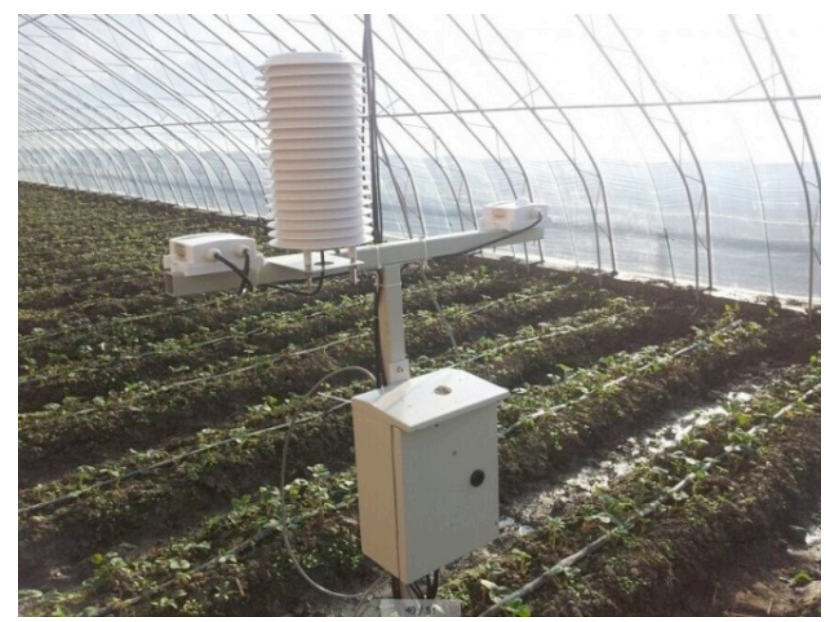

Figure 3. ZigBee convergence node of the physical map nan area, soil is collected from the root of the greenhouse, kept in an aluminum box, dried, and placed in an oven at $105^{\circ} \mathrm{C}$ for $12 \mathrm{~h}$. Soil moisture gradually evaporates. However, the soil structure is not destroyed, and the organic matter is not decomposed. The soil moisture contents of the prepared samples are 5\%,10\%, 20\%, 30\%, 40\%, 50\%, and $60 \%$, which are well modulated for a period. After 6 $\mathrm{h}$, the soil moisture sensor is inserted into the samples for measurement. Each sample measurement is repeated 5 times, and their average value is determined. The relationship between soil water content and the output voltage of the sensor is obtained using the Lagrange interpolation algorithm in numerical analysis.

The calibration system of the temperature and humidity sensor is mainly according to the output voltage value of the sensor. The high-precision temperature and humidity measurement values are compared to determine the corresponding relationship between them. To analyze the sensor calibration results clearly, this study integrates the environmental parameters of the greenhouse WSNmonitoring system into two sensor calibration curves, as shown in Figure 4. The soil water of the greenhouse is shown in Figure 5.

The calibration curve of the sensor can be obtained by analyzing the calibration method. This method can help the system accurately collect the environmental parameters in the greenhouse.

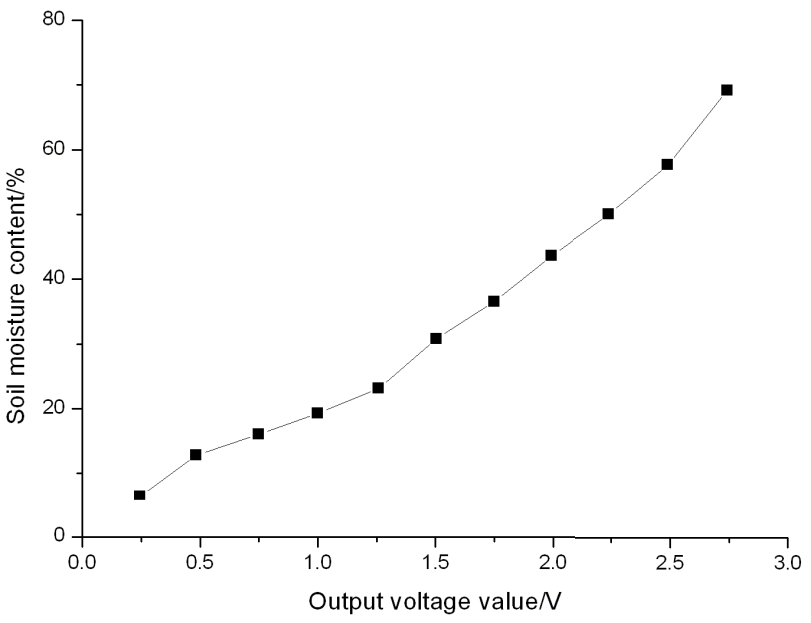

Figure 4. Calibration curve of the sensor

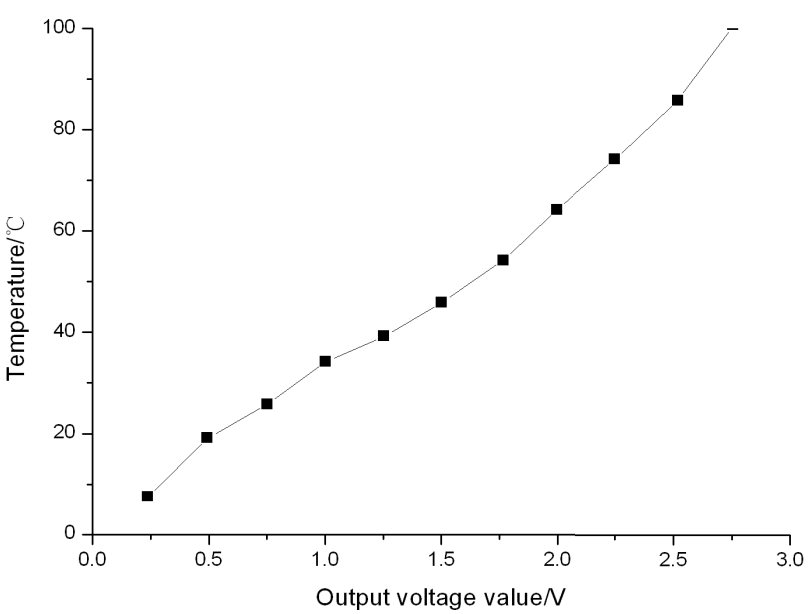

Figure 5. Greenhouse soil water 
The greenhouse-monitoring system should possess network stability, reliability, practicability, stable signal transmission capacity, capacity to work for long hours, high accuracy, anti-jamming capability, and fault tolerance. Therefore, to improve the measurement accuracy of the system, we design the following tests.

The measurement of physical quantities, including greenhouse soil moisture, air temperature, and relative humidity, is conducted according to different measurement parameters and methods to verify the precision. Data acquisition is completed, and the results extracted from the soil moisture sensor placed near the soil area are recorded. The soil-drying method is also conducted in the laboratory. The measurement results of the soil moisture sensor and the traditional soil-drying method are compared. The monitoring accuracy and error are identified.

\section{CONCLUSIONS}

The development of science and technology, communication technology, and sensor technology has provided efficient methods and convenience to many industries. The greenhouse is one of the important symbols of modern agriculture. The combination of WSN applications, modern agriculture, automatic control, computer network, and sensor technologies will considerably help greenhouse development. Greenhouse temperature is detected through WSN, and data are transmitted and analyzed using the new communication technology. This development can effectively reduce the amount of labor required in greenhouse cultivation and improve work efficiency. We evaluate the performance of the entire system and ensure its feasibility and stability. The use of sensor technology significantly helps control the greenhouse environment. With the support of modern technology, agricultural modernization in our country is expected to develop further.

\section{REFERENCES}

[1] Toyosada, Kanako, Takayuki Otani, and Yasutoshi Shimizu. "Water Use Patterns in Vietnamese Hotels: Modeling Toilet and Shower Usage," Water, 2016, vol. 8, no. 3, pp. 85. http://dx.doi.org/10.3390/w8030085

[2] D'Alessandro, Daniela, et al. "Water use and water saving in Italian hospitals. A preliminary investigation," Annali dell'Istituto Superiore di Sanità, 2016, vol. 51, no. 1, pp. 56-62. http://dx.doi.org/10.3390/su8030222

[3] Zhang, Zilong, et al. "The Decoupling of Resource Consumption and Environmental Impact from Economic Growth in China: Spatial Pattern and Temporal Trend," Sustainability, 2016, vol. 8, no. 3, pp. 222.
[4] Kresović, Branka, et al. "Grain yield and water use efficiency of maize as influenced by different irrigation regimes through sprinkler irrigation under temperate climate," Agricultural Water Management, 2016, vol. 169, pp. 34-43. http://dx.doi.org/10.1016/ j.agwat.2016.01.023

[5] Rao, Sajjan Singh, Suresh Pal Singh Tanwar, and Panna Lal Regar. "Effect of deficit irrigation, phosphorous inoculation and cycocel spray on root growth, seed cotton yield and water productivity of drip irrigated cotton in arid environment," Agricultural Water Management, 2016, vol. 169, pp. 14-25. http://dx.doi.org/10.1016/j.agwat.2016.02.008

[6] Wang, Yao-Fen. "Improving Culinary Education by Examining the Green Culinary Behaviors of Hospitality College Students," Journal of Hospitality \& Tourism Education, 2016, vol. 28, no. 1, pp. 1-9. http://dx.doi.org/10.1080/10963758.2015.1127167

[7] González-Cebollada, C. "Water And Energy Consumption After The Modernization Of Irrigation In Spain," WIT Transactions on The Built Environment, 2015, vol. 168, pp. 457-465. http://dx.doi.org/10.2495/SD150401

[8] Xu, Ying, et al. "Improved water management to reduce greenhouse gas emissions in no-till rapeseed-rice rotations in Central China," Agriculture, Ecosystems \& Environment, 2016, vol. 221, pp. 87-98. http://dx.doi.org/10.1016/j.agee.2016.01.021

[9] Shtein, Ilana, Tal Noy-Porat, and Amram Eshel. "Life cycle and reproductive botany of Scilla hyacinthoides, a Mediterranean geophyte," Scientia Horticulturae, 2016, vol. 201, pp. 167-174. http://dx.doi.org/10.1016/j.scienta.2016.01.043

[10] $\mathrm{Xu}, \mathrm{Chi}$, et al. "Effects of Different Irrigation Strategies on Soil Water, Salt, and Nitrate Nitrogen Transport," Ecological Chemistry and Engineering $S$, 2015, vol. 22, no.4, pp. 589-609. http://dx.doi.org/10.1515/eces-2015-0035

\section{AUTHORS}

Yuhong Zhou is with the College of Mechanical and Electrical Engineering, Agricultural University of Hebei, 071001, Baoding, Hebei, China (e-mail: 45446900@qq.com).

Yunfang Xie is with the College of Mechanical and Electrical Engineering, Agricultural University of Hebei, 071001, Baoding, Hebei, China (e-mail: Yun-fang-Xie @126.com).

Limin Shao (corresponding author) is with the College of Mechanical and Electrical Engineering, Agricultural University of Hebei, 071001, Baoding, Hebei, China (e-mail: shaolm@126.com).

This work was supported by Baoding Science and Technology Research and Development Project (14ZG004, 11ZG029) and Polytechnic Foundation of Agricultural University of Hebei (LG20140203). Submitted, 29 March 2016. Published as resubmitted by the authors on 29 April 2016 